\title{
An atypically distributed fleck case with multiple retinal pigment epithelial detachments
}

\begin{abstract}
A 45-year-old female patient presented with complaints of blurred vision and metamorphopsia with a duration of two months. Visual acuities were 0.1 in the right eye and 1.0 in the left eye. Fundus examination revealed bilateral, subretinal, round and yellowwhite flecks with scattered localization in the fovea, macula and peripheral retina. Flecks were mostly concentrated in the macula and midperipheral fundus however were rare in the peripheral fundus. In fluorescein angiography, flecks were stained as well-demarcated hyperfluorescent lesions. In optical coherence tomography, there were hump-shaped lesions which have medium degree internal reflectivity at the level of retinal pigment epithelium along with hyporeflective dome-shaped pigment epithelial detachments. No genetic predisposition was found. As a consequence of these findings, the patient was diagnosed with an atypically distributed fleck case with multiple retinal pigment epithelial detachments.
\end{abstract}

Volume II Issue I - 202I

\author{
Sercan Cate,' Caglar Bektas, ${ }^{2}$ Burak Turgut' \\ 'Department of Ophthalmology, Canakkale On Sekiz Mart \\ University Hospital,Turkey \\ ${ }^{2}$ Department of Ophthalmology, Canakkale Can State Hospital, \\ Turkey
}

\begin{abstract}
Correspondence: Caglar Bektas, Department of Ophthalmology, Canakkale Can State Hospital,Address: Can State Hospital, Can, 17400, Canakkale, Turkey, Tel +90 5555854 l 25, Email drcaglarbektas@gmail.com
\end{abstract}

Received: February 01, 202I | Published: February 15, 2021

Keywords: undefined atypical fleck, pigment epithelial detachment

\section{Introduction}

Flecked retina syndromes, which was first defined by Krill and Klien in 1965, was used for a group of diseases in which yellow or white deep retinal lesions of different sizes and configurations without vascular pathology and optic disc pathology were seen in a limited region or entire fundus. ${ }^{1,2}$ Although it was first used for a group of diseases that included fundus flavimaculatus, fundus albipunctatus, familial drusen and fleck retina of Kandori, many other diseases such as retinitis punctata albescens, Bietti's crystalline dystrophy, and Kjellin's syndrome were added to this group. ${ }^{1-3}$ Diseases in which retinal flecks are observed can be isolated or they can also be seen as a part of a systemic disease such as Alport's syndrome, Sjogren-Larsson syndrome, pseudoxanthoma elasticum, cystinosis, oxalosis, membrano-proliferative glomerulonephritis and vitamin A deficiency. ${ }^{2-5}$

\section{Case report}

A 45-year-old female patient, who had been followed up with a diagnosis of age-related macular degeneration for two years in another hospital, presented with complaints of blurred vision and metamorphopsia, more severely in her right eye, which she said to have started two months ago. The best corrected visual acuities were 0.1 in the right eye and 1.0 in the left eye with Snellen chart. There was no color vision deficiency. There was no complaint of nyctalopia, photosensitivity, or hemeralopia. Visual acuities in light and dark environments were equal. There was no pathology in anterior segment examinations and intraocular pressures were $16 \mathrm{mmHg}$ and $15 \mathrm{mmHg}$ in the right and left eye, respectively. Fundus examination revealed bilateral, subretinal, round and yellow-white flecks with scattered localizations in the fovea, macula and peripheral retina. The diameter of the lesions was approximately one to two times the diameter of the retinal artery. Some of the lesions were merged with each other. Flecks were mostly concentrated in the macula and midperipheral fundus however were rare in the peripheral fundus. Optic nerve heads and retinal vessels were normal, and there were no signs of ocular inflammation (Figure 1). In fundus fluorescein angiography, flecks showed hyperfluorescent staining with welldemarcated borders in early and late phases and there were no signs of choroidal neovascularization or leakage (Figure 2). In optical coherence tomography, there were hump-shaped lesions which have medium degree internal reflectivity at the level of retinal pigment epithelium along with hyporeflective dome-shaped pigment epithelial detachments. No subretinal fluid was observed (Figure 3). There was not any visual field defect. The patient had no other systemic diseases and was not on any medication. There was no family history of retinal pathology. No pathology was found in the ophthalmological and systemic examination of her first-degree relatives. The patient was consulted for genetic diseases, and no genetic disease was found. With these findings, the patient was diagnosed with an atypically distributed fleck case with multiple retinal pigment epithelial detachments and no treatment was recommended. No progression was detected and visual acuities were stable during the 12-month follow-up.

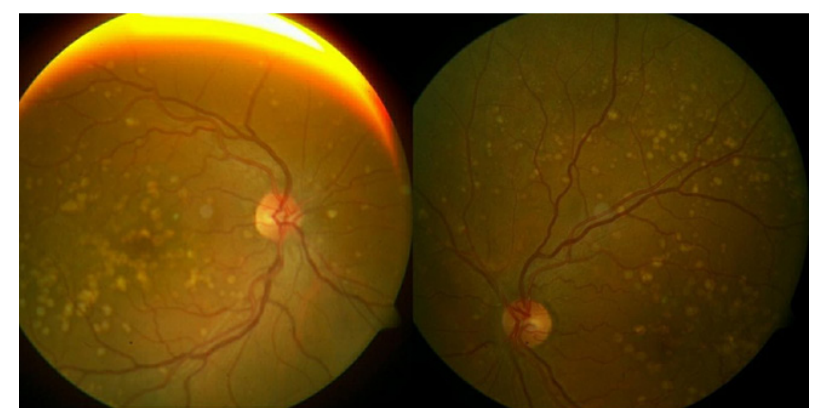

Figure I Bilateral, sub retinal, round and yellow-white flecks with scattered localizations in the fovea, macula and peripheral retina. 


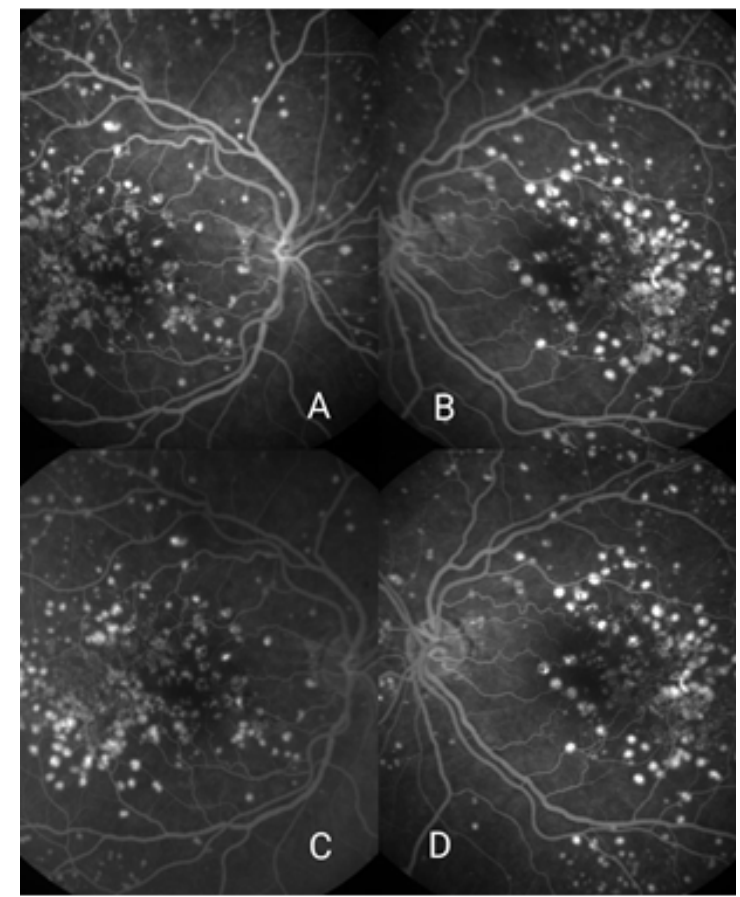

Figure 2 Hyper fluorescent staining with well-demarcated borders in early (A and $B)$ and late $(C$ and $D)$ phases.

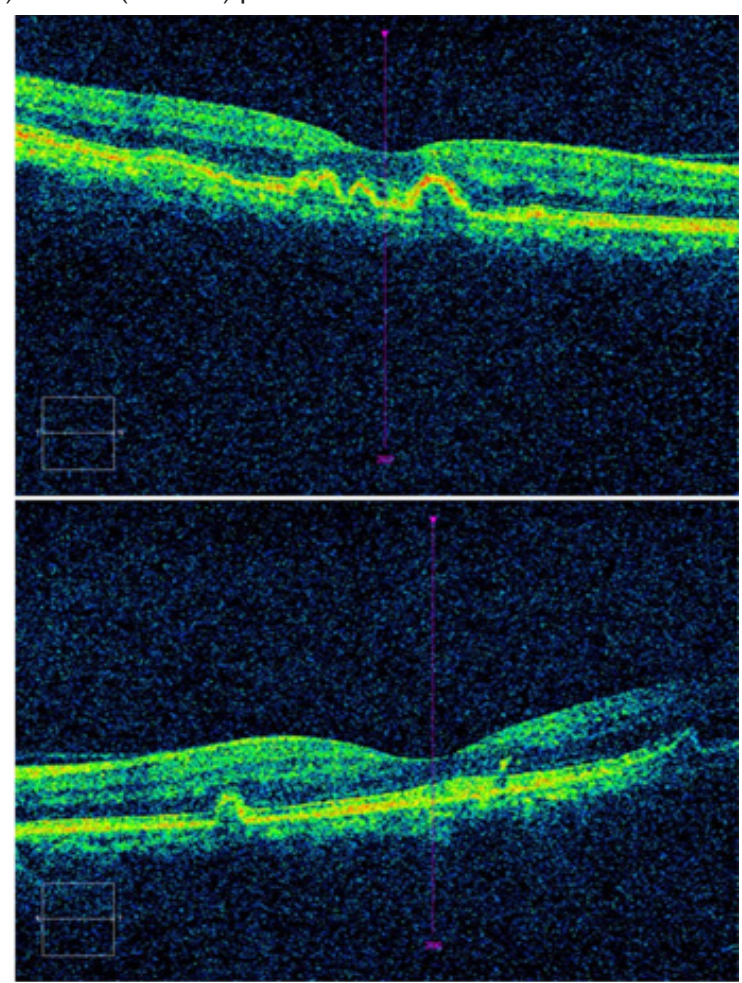

Figure 3 Hump-shaped lesions which have medium degree internal reflectivity at the level of retinal pigment epithelium along with hypo reflective domeshaped pigment epithelial detachments.

\section{Discussion}

In this study, we report a case of late-onset, central and peripherallocated flecks accompanied by multiple retinal pigment epithelial detachments. Retinal flecks can be seen in several diseases with different characteristics. ${ }^{2,3}$
Stargardt's disease is a condition that usually begins in the second decade in which macular atrophy and retinal flecks that spread to the posterior pole are seen. Fundus flavimaculatus is the phenotype of Stargardt's disease in which flecks are distributed in the fundus. ${ }^{6,7}$ The case we report differs from Stargardt's disease in that it has a late onset and does not have macular atrophy. Benign fleck retina is an asymptomatic disease in which diffuse flecks spread to the peripheral retina with a distinctive appearance but the fovea is spared. ${ }^{8,9}$ Our case was distinguished from benign fleck retina in that it is symptomatic and has a different fleck spread pattern. Fundus albipunctatus and retinitis punctata albescens, which are among other flecked retinal disorders, differ from the case we report in terms of causing nyctalopia and sparing fovea. ${ }^{10,11}$

Macular atrophy and retinal pigment epithelium atrophy can be seen in flecked retinal diseases., ${ }^{2,12,13}$ Retinal pigment epithelial detachments may also accompany flecked retinal diseases, as seen in our case and in the case of Garcia et al. ${ }^{9}$ Because retinal flecks can be confused with drusen, and both retinal atrophy and pigment epithelial detachment can be seen in flecked retinal diseases and agerelated macular degeneration, it may lead to incorrect diagnosis. In this respect, it is important to distinguish flecks from drusen. Drusen, which are the typical lesion of age-related macular degeneration, are seen as lesions of varying sizes located between the retinal pigment epithelium and Bruch's membrane. ${ }^{14}$ On the contrary, flecks begin at the level of the retinal pigment epithelium and migrate to the outer retinal layers over time..$^{13,15}$ Large colloid drusen which is a subgroup of early onset drusen was recently described by Guigui et al. Although our case and large colloid drusen show similarity with distributions of lesions and presence of pigment epithelial detachments, they have different staining patterns in the early phase of fluorescein angiography. ${ }^{16,17}$

Flecked retinal disorders are usually autosomal recessive diseases, and family screening is therefore important. They may also be as a part of some syndromes, such as Alport syndrome, Sjogren-Larsson syndrome, pseudoxanthoma elasticum. ${ }^{2}$ Our case was screened for systemic and genetic disorders; however no accompanying pathology was found. First-degree relatives were scanned in terms of ocular and systemic diseases, and no ocular and systemic pathology was found. Therefore, the case was evaluated as an isolated flecked retinal disease.

\section{Conclusion}

In conclusion, the presented case was evaluated as an atypical and educative case since it has different clinical features from reported flecked diseases and can easily be confused with age-related macular degeneration due to the accompanying pigment epithelial detachment.

\section{Acknowledgments}

None.

\section{Conflicts of interest}

There are no conflicts of interest.

\section{Funding}

None.

\section{References}

1. Krill AE, Klien BA. Flecked retina syndrome. Arch Ophthalmol.1965;74:496-508. 
2. Walia S, Fishman GA, Kapur R. Flecked-retina syndromes. Ophthalmic genetics. 2009;30(2):69-75.

3. Laey JJ. Flecked retina disorders. Bulletin de la Société belge d'ophtalmologie. 1993;249:11-22.

4. Colville DJ, Savige J. Alport syndrome. A review of the ocular manifestations. Ophthalmic Genet. 1997;18(4):161-173.

5. Finger RP, Charbel Issa P, Ladewig MS, et al. Pseudoxanthoma elasticum: genetics, clinical manifestations and therapeutic approaches. Surv Ophthalmol. 2009;54(2):272-285.

6. Fishman GA. Fundus flavimaculatus. A clinical classification. Arch Ophthalmol. 1976;94(12):2061-2067.

7. Gupta CP. Fundus flavimaculatus. Br J of Ophthalmol. 1973;57:439-440.

8. Sabel Aish SF, Dajani B. Benign familial fleck retina. Br J Ophthalmol. 1980’64(9):652-659.

9. Garcia JMBB, Isaac DLC, Sardeiro T, et al. Benign familial fleck retina: multimodal imaging including optical coherence tomography angiography. Arq Bras Oftalmol. 2017;80(5):321-323.

10. Sergouniotis PI, Sohn EH, Li Z, et al. Phenotypic variability in RDH5 retinopathy (Fundus Albipunctatus). Ophthalmology. 2011;118(8):16611670 .
11. Fishman GA, Roberts MF, Derlacki DJ, et al. Novel mutations in the cellular retinaldehyde-binding protein gene (RLBP1) associated with retinitis punctata albescens: evidence of interfamilial genetic heterogeneity and fundus changes in heterozygotes. Archives of Ophthalmology (Chicago, Ill. : 1960). 2004;122(1):70-75.

12. Pichi F, Abboud EB, Ghazi NG, et al. Fundus autofluorescence imaging in hereditary retinal diseases. Acta Ophthalmol. 2018;96(5):e549-e561.

13. Querques G, Leveziel N, Benhamou N, et al. Analysis of retinal flecks in fundus flavimaculatus using optical coherence tomography. $\mathrm{Br} J$ Ophthalmol. 2006;90(9):1157-1162.

14. Chen Y, Vuong LN, Liu J, et al. Three-dimensional ultrahigh resolution optical coherence tomography imaging of age-related macular degeneration. Opt Express. 2009;17(5):4046-4060.

15. Solberg Y, Dysli C, Escher P, et al. Retinal flecks in Stargardt disease reveal characteristic fluorescence lifetime transition over time. Retina. 2019;39(5):879-888.

16. Guigui B, Leveziel N, Martinet V, et al. Angiography features of early onset drusen. Br J Ophthalmol. 2011;95(2):238-244.

17. Guigui B, Querques G, Leveziel N, et al. Spectral-domain optical coherence tomography of early onset large colloid drusen. Retina. 2013;33(7):1346-1350. 